Hautarzt 2016 $67: 780-785$

DOI 10.1007/s00105-016-3866-3

Online publiziert: 31. August 2016

(C) Der/die Autor(en) 2016. Dieser Artikel ist eine Open-Access-Publikation.

CrossMark

\author{
I. Schwarz · D. Bokanovic · W. Aberer \\ Univ. Klinik für Dermatologie, LKH Graz, Graz, Österreich
}

\title{
Schleimhauterkrankungen aus allergologischer Sicht
}

(SJS) oder der toxischen epidermalen Nekrolyse (TEN).

der Schleimhaut sind häufig. Die
dergische Erkrankungen an Schleimhäute im Augenbereich und Respirationstrakt sind gegenüber inhalativen Allergenen exponiert. Am Gastrointestinaltrakt manifestieren sich insbesondere Symptome der Nahrungsmittelallergie, wobei die Schleimhäute des MundRachen-Magen-Darm-Traktes als eine der größten Körperbarrieren zur Außenwelt prädestiniert für die Entwicklung allergischer Reaktionen sind. In diesem Beitrag soll das Hauptaugenmerk auf die allergischen Kontaktreaktionen an der Mund- und Anogenitalschleimhaut gelegt werden.

An der oralen und anogenitalen Schleimhaut treten allergische Soforttyp- und Spättypreaktionen mit unterschiedlicher Häufigkeit auf. Zur Ursachenklärung ist eine genaue Anamneseerhebung bezüglich spezifischer Expositionsfaktoren unerlässlich. Allergische Reaktionen an der Mundschleimhaut können im Sinne einer Soforttypreaktion als orales Allergiesyndrom auftreten oder sich auch als Spättypreaktion in Form einer Kontaktstomatitis oder Kontaktcheilitis manifestieren. Auch bei Angioödemen ist aufgrund des lockeren Bindegewebes die Mundschleimhaut häufig betroffen. An der Anogenitalschleimhaut treten Kontaktekzeme wesentlich häufiger auf als Soforttypreaktionen; erwähnt seien hier die Kontakturtikaria auf Seminalplasma oder Naturlatex. Reaktionen auf Arzneimittel manifestieren sich an der Mund- und Genitalschleimhaut unter anderem als fixes Arzneiexanthem oder auch mit wesentlich gravierenderer Affektion beim Stevens-Johnson-Syndrom

\section{Allergische Kontakt- reaktionen im Bereich der Mundschleimhaut}

\section{Orales Allergiesyndrom}

Beim oralen Allergiesyndrom (OAS) handelt es sich um eine IgE-vermittelte Nahrungsmittelallergie, bei der es bereits wenige Minuten nach dem Verzehr roher Obst- und Gemüsesorten oder takturtikaria der Mundschleimhaut mit meist milden oropharyngealen Symptomen kommt. Ursächlich handelt es sich zumeist um eine sekundäre Nahrungsmittelallergie im Rahmen einer Kreuzallergie mit Pollenallergenen (z. B. Bet v 1-homologe Allergene, Profiline) [27]. Die primäre Sensibilisierung erfolgt über die Atemwege [30]. Im Gegensatz dazu ist bei der primären Nahrungsmittelallergie das Nahrungsmittelprotein selbst Ursache der Sensibilisierung. Primäre Nahrungsmittelallergien führen außerdem häufiger zu systemischen allergischen Reaktionen [21].

Die Symptome des OAS treten für gewöhnlich unmittelbar bei Kontakt zum allergenen Nahrungsmittel auf, seltener mit einer Latenz bis zu $2 \mathrm{~h} \mathrm{[30].} \mathrm{Ab-}$ gesehen vom Jucken oder Kribbeln der Lippen und des Mundes wird auch über orales Brennen, Schwellungen oder sogar ein laryngeales Engegefühl berichtet [21]. Fallweise entwickeln sich auch Erytheme oder kleine Vesikel an der Mundschleimhaut (insbesondere labial) [30]. Die Beschwerden können während der Pollensaison verstärkt auftreten. Abhängig von Prozessierung, zugeführter Menschon währenddessen zu einer Kon- ge und Matrixeffekten können bestimmte pollenassoziierte Nahrungsmittelallergene (z. B. von Sellerie oder Soja) fallweise auch systemische Anaphylaxiesymptome auslösen ([27]; • Tab. 1).

Die Diagnose einer pollenassoziierten Nahrungsmittelallergie beruht auf der Anamneseerhebung, dem Nachweis einer Sensibilisierung (Hauttestung und/oder serologische IgE-Bestimmung) und der oralen Provokationstestung. Die Hauttestung sollte mit inhalativen Allergenen und mit deren assoziierten Nahrungsmittelallergenen erfolgen. Ein Pricktest mit nativen, $d . h$. frischen Nahrungsmitteln hat übrigens eine deutlich höhere Sensitivität als der Test mit kommerziellen Nahrungsmitteltestlösungen. Um die klinische Relevanz einer mittels Haut- oder In-vitro-Testung nachgewiesenen Sensibilisierung zu prüfen, ist allerdings die positive Anamnese ausschlaggebend. Bei unklarer Anamnese, einschließlich eines unklaren Ernährungs- und Symptomenprotokolls, kann nur der orale Provokationstest eine klinisch relevante Nahrungsmittelallergie von einer stummen Sensibilisierung abgrenzen [30].

\section{》) Die Allergenkarenz ist die sicherste therapeutische Maßnahme}

Bei Nachweis einer klinisch relevanten Kreuzreaktion stellt die Allergenkarenz die sicherste therapeutische Maßnahme dar. Die Durchführung einer spezifischen Immuntherapie kann in Einzelfällen wirksam sein, die Studienlage hierzu ist jedoch nicht einheitlich. Aus diesem Grund sollte diese allerdings nur bei Patienten mit gleichzeitiger allergischer 
Tab. 1 Pollenassoziierte Nahrungsallergene, die häufiger ein orales Allergiesyndrom auslösen. (Mod. nach [27])

\begin{tabular}{|c|c|c|c|}
\hline & $\begin{array}{l}\text { Birke } \\
\text { Erle } \\
\text { Hasel }\end{array}$ & Gräser & Beifuß \\
\hline Baumnüsse: Haselnuss, Walnuss, Paranuss, Mandel & +++ & - & + \\
\hline Kernobst: Apfel, Birne & +++ & + & - \\
\hline Steinobst: Pflaume, Pfirsich, Kirsche, Aprikose & +++ & + & - \\
\hline Karotte & ++ & - & ++ \\
\hline Tomate & ++ & + & + \\
\hline Soja & +++ & + & - \\
\hline Erdnuss & + & + & - \\
\hline Sellerie & ++ & + & +++ \\
\hline Gewürze: Anis, Dill, Petersilie, Pfeffer, Paprika, Kümmel & + & - & +++ \\
\hline
\end{tabular}

Rhinokonjunktivitis erfolgen. Ein orales Allergiesyndrom ohne begleitende respiratorische Symptome stellt keine Indikation für die spezifische Immuntherapie dar $[11,30]$.

\section{Allergische Kontaktstomatitis und Kontaktcheilitis}

Kontaktallergische Spättypreaktionen an der Mundschleimhaut treten sehrviel seltener als an der Haut auf. Eine mögliche Ursache ist beispielsweise die sehr viel seltenere bzw. weniger intensive Exposition gegenüber potenziellen Kontaktallergenen [6].

Die häufigsten Reaktionen gegenüber den auslösenden Kontaktstoffen sind Rötung, Schwellung, gelegentlich auch Blasenbildung, Erosionen oder Ulzerationen. Auch lichenoide Reaktionen im Sinne einer oralen lichenoiden kontaktallergischen Reaktion können vorkommen. Diese zeigt, ähnlich wie der orale Lichen ruber, eine weißliche Netzstreifung, Erytheme oder auch Erosionen an der bukkalen und gingivalen Mukosa. Sie ist von diesem aber als eine immunologische Hypersensitivitätsreaktion auf spezifische Auslöser abzugrenzen [4].

Kontaktcheilitiden können auch ohne begleitende Kontaktstomatitis auftreten und zeichnen sich durch Fissuren und Schuppung, bei stärkerer Ausprägung durch Erytheme, Ödeme, Bläschen und Krusten aus [6]. Nach exakter Anamneseerhebung sollte zur Diagnosestellung der Nachweis einer Sensibilisierung mittels Epikutantestung erfolgen. Ein epimuköser Test ist nicht notwendig da eine klinisch relevante Kontaktallergie der Mundschleimhaut auch an der Haut nachweisbar ist [27].

Häufige relevante Kontaktallergene bei Kontaktstomatitiden sind Zahnersatzmaterialien und zahnärztliche Werkstoffe wie dentale Adhäsionsmaterialien (Acrylate, Eugenol), Dentalmetalle (Kobalt, Kupfer, Chrom, Gold, Quecksilber, Nickel, Silber), Komposit und Porzellan. Des Weiteren können die in Dentalmaterialien enthaltenen Duftstoffe Perubalsam, Zimt, Eugenol, Pfefferminze und Menthol sowie das Konservierungsmittel Dodecylgallat relevant sein $[4,22,26]$. Die klinische Relevanz eines positiven Tests auf ein ubiquitäres Allergen wie Nickel ist allerdings oft schwer beweisbar. Bei Patienten mit Zahnprothesen und brennenden oralen Schmerzen sollte vor Durchführung einer aufwendigen allergologischen Abklärung auch an eine orale Candidiasis gedacht und diese entsprechend abgeklärt und therapiert werden [23].

Potenzielle Kontaktallergene als Auslöser von Kontaktcheilitiden sind unter anderem diverse Externa, wie z. B. Herpessalben (Tromantadin, Idoxuridin, Trifluridin, Aciclovir, Penviclovir), Lippenpflegeprodukte, Kosmetika (Lippenstift), des Weiteren Duftstoffe, Nagellack (Autotransfer durch Nagelkauen), Nickel und Gummi („nervöses“ Kauen auf entsprechenden Gegenständen) [27].

\section{Allergische Kontakt- reaktionen im Bereich der Anogenitalschleimhaut}

Der Anogenitalbereich ist durch die Verwendung von Hygieneartikeln, Pflegeprodukten, topischen Medikamenten sowie Verhütungsmethoden einer Vielzahl von Allergenen ausgesetzt. Aufgrund von Schamgefühl berichten Patienten nur ungern über ihre genitalen Beschwerden, weshalb allergische Erkrankungen in diesem Bereich häufig unterdiagnostiziert sind. Insgesamt kommen Spättypreaktionen im Anogenitalbereich wesentlich häufiger vor als Soforttypreaktionen, wie z. B. eine Kontakturtikaria auf LatexKondome oder im Rahmen einer Spermaallergie.

\section{Kontaktekzeme der Anogenital- schleimhaut}

Anogenitaldermatosen sind insgesamt zwar häufige Krankheitsbilder, allerdings selten allergischer Genese. Retrospektive Auswertungen zeigen, dass im Rahmen der Abklärung von Patienten mit der Zuweisungsdiagnose „Anogenitaldermatose" nur in etwa $25 \%$ der Fälle die Diagnose eines allergischen Kontaktekzems gestellt werden konnte. Bei etwa $12 \%$ wurde eine chronisch irritative Dermatitis diagnostiziert [13]. Häufig handelt es sich auch um eine banale Intertrigo oder Candidiasis [12].

\section{》) Die allergische Kontaktder- matitis tritt mit einer Latenz von 24-48 h nach Allergenkontakt auf}

Ein allergisches Kontaktekzem ist von einem irritativen oft schwer zu unterscheiden. Während sich die irritative Kontaktdermatitis innerhalb von Minuten nach Exposition entwickelt, tritt die allergische Kontaktdermatitis für gewöhnlich erst mit einer Latenz von 24-48 h nach Allergenkontakt auf. Symptome wie brennende Schmerzen stehen eher bei der irritativen Dermatitis im Vordergrund, Juckreiz bei der allergischen Kontaktdermatitis. Bei beiden Formen zeigen sich Erytheme, 
Ödeme, evtl. konfluierende Bläschen und nässende Blasen. Die sonst für die chronische Kontaktdermatitis typische Krustenbildung kommt an der Schleimhaut aufgrund des hier herrschenden feuchten okklusiven Milieus nicht vor [3].

Zur diagnostischen Abklärung zählen neben einer exakten Anamneseerhebung und der klinischen Inspektion eine Epikutantestung einschließlich der von den Patienten verwendeten Externa [12]. Häufige Ursachen von Kontaktdermatitiden im Anogenitalbereich sind Wirkstoffe von Lokaltherapeutika (Hämorrhoidensalben), Duftstoffe, Konservierungsmittel (feuchte Toilettentücher) und Salbengrundlagen [2]. Dagegen scheinen Inhaltsstoffe von Waschmitteln als Ursache für die Entstehung einer Kontaktallergie eine untergeordnete Rolle zu spielen [13].

\section{Kondomallergie}

Eine Allergie gegen Naturlatex als Bestandteil von Kondomen ist selten. $\mathrm{Zu}$ meist handelt es sich um eine Soforttypreaktion gegen die Proteinkontamination im Naturlatex. Diese äußert sich als eine auf die Kontaktstelle beschränkte Kontakturtikaria mit Juckreiz und Schwellung. Es existieren aber auch Fallberichte über Spätreaktionen nach Gebrauch von Kondomen, die sich als schwere Kontaktdermatitiden des Penis bzw. der Vulva und Vagina manifestieren. Diese treten aufgrund von Sensibilisierungen auf Gummizusatzstoffe auf [5].

\section{Spermaallergie}

Die Spermaallergie ist eine sehr seltene IgE-vermittelte Typ-I-Allergie. Die Prävalenz und Inzidenz sind weitgehend unbekannt. Es wird aber davon ausgegangen, dass in den USA bis zu 40.000 Frauen betroffen sind. Sie manifestiert sich als ein Spektrum von lokalen und auch systemischen Reaktionen nach Kontakt mit spezifischen Proteinkomponenten des Seminalplasmas. Hauptallergen ist das prostataspezifische Antigen (PSA). Lokalreaktionen treten unmittelbar bis maximal $1 \mathrm{~h}$ postkoital auf und umfassen vulvovaginales Brennen, Schmerzen, Juckreiz, Rötungen und Schwellungen,

Hautarzt 2016 $67: 780-785 \quad$ DOI 10.1007/s00105-016-3866-3

(c) Der/die Autor(en) 2016. Dieser Artikel ist eine Open-Access-Publikation.

\section{Schwarz $\cdot$ D. Bokanovic $\cdot$ W. Aberer}

Schleimhauterkrankungen aus allergologischer Sicht

\section{Zusammenfassung}

Das orale Allergiesyndrom stellt in unseren Breiten die häufigste Manifestation einer Nahrungsmittelallergie dar und äußert sich nach Verzehr kreuzreaktiver Nahrungsmittel als Kontakturtikaria an der Mundschleimhaut. Während Dentalmaterialien häufige Auslöser von Spättypreaktionen im Sinne von Kontaktstomatitiden sind, treten Kontaktcheilitiden häufig als Reaktion auf verschiedene Externa wie Herpessalben oder Lippenpflegeprodukte auf. An der Anogenitalschleimhaut treten Spättypreaktionen wesentlich häufiger auf als Soforttypreaktionen. Kontaktekzeme in diesem Bereich sollten mittels Epikutantestung abgeklärt werden. Bei Vorliegen einer Kontakturtikaria der Genitalschleimhaut sollte an eine Seminalplasma- oder Latexallergie gedacht werden. Angioödeme, die zumeist histaminvermittelt sind, betreffen bevorzugt Hautareale mit lockerem Bindegewebe wie die Mund-, aber auch die Genitalschleimhaut. Beim fixen Arzneimittelexanthem besteht ebenso eine Prädilektion der Mund- und Genitalschleimhaut. Bullöse Arzneireaktionen wie das Stevens-Johnson Syndrom und die toxische epidermale Nekrolyse sind zumeist durch einen schweren hämorrhagischen, erosiven Schleimhautbefall gekennzeichnet.

\section{Schlüsselwörter}

Nahrungsmittelallergie $\cdot$ Angioödem . Kontaktallergie · Anogenital · Arzneimittelexanthem

\section{Mucosal diseases from an allergological perspective}

\section{Abstract}

The oral allergy syndrome is one of the most common form of food allergy and manifests as contact urticaria of the oral mucosa after consumption of cross reacting foods. Whereas allergic contact stomatitis often occurs due to dental materials, allergic contact cheilitis is usually a reaction due to topical therapeutics like herpes ointments or lip care products. As late type reactions are more frequent than immediate type reactions in the anogenital mucosa, contact dermatitis in this area should be identified via epicutaneous testing. In case of contact urticaria at the genital mucosa, a semen allergy or a latex allergy should be given due consideration as a possible cause. Angioedemas, which are mostly common histamine mediated, usually prefer skin areas with loose connective tissue such as the oral or genital mucosa. Fixed drug eruption also occurs preferentially in these areas. Bullous drug-induced skin reactions (e.g., SJS and TEN) are characterized by severe hemorrhagic, erosive affections of mucous membranes.

\section{Keywords}

Food allergy - Angioedema - Contact sensitization · Anogenital area · Drug eruptions gelegentlich assoziiert mit kleinen Bläschen. Die Symptome können einige Tage bis Wochen persistieren $[1,25]$.

\section{Angioödeme}

Angioödeme sind selbstlimitierende, lokalisierte subkutane oder submuköse Schwellungen. Typischerweise sind Hautareale mit lockerem Bindegewebe wie Gesicht (Augenlider, Wangen, Lippen), Zunge, Larynx, Pharynx, Genitalien, aber auch Extremitäten betroffen [27]. Die Schwellungen persistieren für gewöhnlich 1 bis 3 Tage, manchmal bil- den sie sich bereits nach einigen Stunden oder erst nach 7 Tagen zurück [7].

Das klinische Symptom „Angioödem“ findet sich bei unterschiedlichen Krankheitsentitäten. Am häufigsten sind Angioödeme Teilbild oder Äquivalent einer Urtikaria (• Abb. 1). In diesen Fällen handelt es sich um histaminvermittelte Angioödeme, die im Rahmen allergischer Soforttypreaktionen auf Insektengifte, Medikamente und Nahrungsmittel oder auch idiopathisch auftreten können. Hinsichtlich Pathogenese, Klinik und Therapie unterscheidet man von diesen bradykininvermittelte Angioödeme bei hereditärem oder erworbenem 


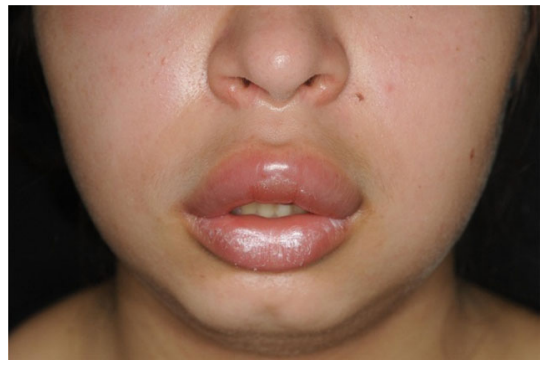

Abb. 1 ॥ Angioödem als Teilbild einer Urtikaria

C1-Inhibitor-Mangel. Das hereditäre Angioödem (HAE) mit normalem C1Inhibitor (HAE Typ III) sowie ACEHemmer-induzierte Angioödeme zählen $\mathrm{zu}$ jenen Angioödemen, deren $\mathrm{Pa}$ thomechanismus noch nicht vollständig geklärt ist $[7,8]$.

\section{I) Bei vital bedrohlicher Atemnot sollte unverzüglich eine Intubation erfolgen}

Bei Angioödemen im Mundbereich sind zumeist Zunge und Lippen betroffen, seltener Uvula und Rachenschleimhaut bzw. Epiglottis. Begleitsymptome eines beginnenden Glottisödems sind Tieferwerden der Stimme, Kloßgefühl und Schluckbeschwerden sowie schließlich Atemnot und Erstickungsgefühl [6]. Die medikamentöse Therapie ergibt sich aus der $\mathrm{Pa}$ thogenese und dem Schweregrad des Angioödems. Primär sollten $\mathrm{H}_{1}$-Antihistaminika peroral verabreicht werden, bedarfsweise bis zum 4-Fachen der empfohlenen Dosierung. Bei unzureichender Symptomkontrolle kann die medikamentöse Therapie durch eine orale Kortisonstoßtherapie (bis zu $50 \mathrm{mg}$ Pred- nisolonäquivalent pro Tag über maximal 10 Tage) erweitert werden. Angioödeme im Kopfbereich mit Ödem des Pharynx oder Larynx sollten aufgrund der drohenden Erstickungsgefahr stationär mit intravenöser Verabreichung von $\mathrm{H}_{1}$-Antihistaminika und Kortikosteroiden (bis zu 100-250 mg Prednisolonäquivalent) behandelt und überwacht werden. Bei vital bedrohlicher Atemnot sollte unverzüglich eine Intubation erfolgen. Treten weitere Symptome auf, die eine Anaphylaxie wahrscheinlich machen, ist die übliche Anaphylaxiebehandlung einzuleiten [7, 16, 29, 31].

Das hereditäre bradykininvermittelte Angioödem spricht nicht auf die Gabe von Antihistaminika oder Kortikosteroiden an. Es kann jedoch klinisch nicht von einem histaminvermittelten Angioödem unterschieden werden. Da histaminvermittelte Angioödeme weit häufiger sind, ist bei fehlender Diagnose die Therapie mit Antihistaminika und Kortikosteroiden indiziert (s.oben) [9]. Für die Therapie akuter Anfälle des hereditären Angioödems stehen in EU-Ländern folgende Präparate zur Verfügung: C1-Inhibitor-Konzentrate aus humanem Plasma (Berinert ${ }^{\circledR}$, Cinryze $\left.^{\circledR}\right)$, rekombinanter humaner C1-Inhibitor (Ruconest ${ }^{\circledR}$ ), der Bradykininrezeptorantagonist Icatibant $\left(\right.$ Firazyr $^{\circledR}$ ) oder gefrorenes Frischplasma. Die zur Verfügung stehenden Präparate sollten unverzüglich nach Auftreten von Symptomen verabreicht werden. Aus diesem Grund besteht inzwischen die Möglichkeit, Patienten in der Selbstadministration zu schulen. In der EU sind zu diesem Zweck folgende Präparate zugelassen: Berinert ${ }^{\circledR}$, Cinryze $^{\circledR}$ (intravenös) und Firazyr ${ }^{\circledR}$ (subkutan) [8].

\section{Arzneimittelreaktionen mit Schleimhautbeteiligung}

\section{Fixes Arzneimittelexanthem}

Beim fixen Arzneimittelexanthem handelt es sich um rezidivierende Hautund/oder Schleimhautläsionen, die nach wiederholter Aufnahme des auslösenden Arzneimittels immer an denselben Stellen auftreten. Die Symptome treten etwa $30 \mathrm{~min}$ bis $8 \mathrm{~h}$ nach Einnahme des auslösenden Medikaments auf [18]. Pathogenetisch handelt es sich um eine durch systemische Gabe von Arzneimitteln induzierte Spättypreaktion. Es konnte gezeigt werden, dass $\mathrm{CD}^{+}-\mathrm{T}$ Gedächtniszellen, die an den Lokalisationen der fixen Arzneireaktion intraepidermal persistieren, eine zentrale Rolle beim neuerlichen Auftreten der Läsionen spielen $[10,18]$. Die Genitalschleimhaut ist mit etwa 50 \% der Fälle am häufigsten betroffen, gefolgt von Stamm, Lippen und Händen [17].

An der oralen Schleimhautmukosa präsentiert sich das fixe Arzneimittelexanthem häufig bullös, erosiv, aphthös oder erythematös. Es ist meistens am harten Gaumen und am Zungenrücken lokalisiert. Die Läsionen heilen für gewöhnlich ohne Restpigmentierung ab [19]. An der Genitalschleimhaut zeigen sich solitäre oder multilokuläre, scharf begrenzte münz- bis handtellergroße, runde oder ovale Erytheme, ödematöse lividrote Plaques, gelegentlich Blasen und Erosionen [27]. Bei Männern tritt das fixe Arzneimittelexanthem bevorzugt an der Glans penis und am Sulcus coronarius auf $[10,20]$.

Hier steht eine Anzeige. 
Als häufigste auslösende Arzneimittel gelten Cotrimoxazol (Trimethoprim, Sulfamethoxazol), Naproxen, Oxicame, Tetrazykline und Acetylsalicylsäure. Naproxen und Oxicame verursachen am häufigsten Läsionen an den Lippen, Cotrimoxazol an der Genitalschleimhaut [17].

\section{Generalisierte Arzneireaktionen mit Schleimhautbeteiligung}

Bei der Ätiologie der generalisierten bullösen Arzneireaktionen spielen neben Arzneimitteln auch häufig Infektionskrankheiten eine Rolle. Während das Erythema exsudativum multiforme (EEM) häufig mit Infektionen wie Herpes simplex, Mykoplasmen oder Streptokokken assoziiert ist, stehen beim SJS und der TEN Medikamente als Auslöser im Vordergrund. Häufige medikamentöse Auslöser eines SJS oder einer TEN sind Allopurinol, Sulfonamide, Antikonvulsiva wie Carbamazepin, Phenytoin und Phenobarbital, nichtsteroidale Antirheumatika (NSAR) vom Oxicam-Typ sowie das antiretrovirale Therapeutikum Nevirapin [27]. Pathogenetisch werden die Hautreaktionen als T-Zell-vermittelte Reaktionen angesehen, da in der Dermis überwiegend $\mathrm{CD}^{+}$-Zellen und in der Epidermis $\mathrm{CD}^{+}$-Zellen nachgewiesen wurden [14].

Während es bei der TEN per definitionem nahezu immer zu einem Schleimhautbefall kommt, zeigt sich beim SJS in $92-100 \%$ und beim EEM nur in etwa $25-60 \%$ der Fälle eine Schleimhautbeteiligung [24]. Bezüglich der Intensität der Mundschleimhaut- und Konjunktivalbeteiligung bestehen keine signifikanten Unterschiede bei EEM, SJS und TEN. An der Mundschleimhaut können sich erosive Cheilitiden und Stomatitiden sowie hämorrhagische Krusten zeigen, an den Konjunktiven Konjunktivitiden, Erosionen und Synechien. Eine Beteiligung der Genitalschleimhaut äußert sich als Vulvitis oder Balanitis. Im Rahmen der TEN können sogar Atemwege und Magen-Darm-Trakt in Form von Tracheobronchitis, Ösophagitis, Gastritis, Enterokolitis und Proktitis beteiligt sein [27].
Die Letalität ist abhängig vom Ausmaß der Blasenbildung, dem Alter der Patienten und den bestehenden Grunderkrankungen und liegt beim SJS bei $9 \%$ und bei der TEN bei $48 \%$ [15].

\section{\) SJS und TEN gelten als Notfälle und bedürfen einer Intensivbetreuung}

Bei Verdacht auf Vorliegen einer schweren generalisierten Arzneireaktion sollten nach exakter Anamneseerhebung alle als Auslöser infrage kommenden Medikamente sofort abgesetzt und es sollte ein auf Arzneireaktionen spezialisiertes Zentrum kontaktiert werden. SIS und TEN gelten als Notfälle und bedürfen einer Intensivbetreuung. Die Gabe von immunmodulierenden Substanzen wie intravenösen Immunglobulinen (IVIG) und Glukokortikosteroiden wird kontrovers diskutiert [14]. Studien unter kontrollierten Bedingungen fehlen, und es gibt - teils heftige - Pro- und Kontra-Argumente. Eine einzige, allerdings auch offene Studie lässt den Schluss $\mathrm{zu}$, dass Ciclosporin die Todesrate bei diesen Krankheitsbildern senkt und die Progression der Hautablösung positiv beeinflussen kann [28]. Stellt allerdings eine allergische Reaktion, etwa auf ein Arzneimittel, aufgrund des zeitlichen Ablaufs den recht sicheren Auslöser dar, wäre dies wohl ein sehr starkes Argument für den schnellen und hoch dosierten Einsatz von Glukokortikosteroiden.

\section{Fazit für die Praxis}

- Beim oralen Allergiesyndrom sollte die Durchführung einer spezifischen Immuntherapie zur Symptommilderung nur bei Patienten mit gleichzeitig bestehenden Beschwerden einer Rhinokonjunktivitis in Betracht gezogen werden.

- Angioödeme im Kopfbereich mit Pharynx- und/oder Larynxödem sollten aufgrund der drohenden Erstickungsgefahr stationär behandelt und überwacht werden.

- Bei Auftreten einer Kontakturtikaria im Genitalbereich nach geschütz- tem Geschlechtsverkehr ist an eine Latexallergie zu denken.

- Bei Anogenitaldermatosen sollte ein allergisches Kontaktekzem mittels Epikutantestung ausgeschlossen werden.

- Bei Vorliegen einer Kontaktcheilitis sollte auch an einen möglichen Autotransfer von Allergenen durch $\mathrm{Na}$ gelkauen (Nagellack) und „nervöses” Kauen auf bestimmten Gegenständen gedacht werden.

- Bei Verdacht auf Vorliegen einer schweren generalisierten Arzneireaktion sollten nach exakter Anamneseerhebung alle als Auslöser infrage kommenden Medikamente sofort abgesetzt und es sollte ein auf Arzneireaktionen spezialisiertes Zentrum kontaktiert werden.

\section{Korrespondenzadresse}

\section{Dr. I. Schwarz}

Univ. Klinik für Dermatologie, LKH Graz Auenbruggerplatz 8, $8036 \mathrm{Graz}$, Österreich ines.schwarz@medunigraz.at

Open access funding provided by Medical University of Graz.

\section{Einhaltung ethischer Richtlinien}

Interessenkonflikt. I. Schwarz, D. Bokanovic und W. Aberer geben an, dass kein Interessenkonflikt besteht.

Dieser Beitrag beinhaltet keine von den Autoren durchgeführten Studien an Menschen oder Tieren.

Open Access. Dieser Artikel wird unter der Creative Commons Namensnennung 4.0 International Lizenz (http://creativecommons.org/licenses/by/4.0/deed. de) veröffentlicht, welche die Nutzung, Vervielfältigung, Bearbeitung, Verbreitung und Wiedergabe in jeglichem Medium und Format erlaubt, sofern Sie den/die ursprünglichen Autor(en) und die Quelle ordnungsgemäßnennen, einen Link zur Creative Commons Lizenz beifügen und angeben, ob Änderungen vorgenommen wurden.

\section{Literatur}

1. Allam JP, Haidl G, Novak N (2015) Semen allergy. Hautarzt 66:919-923

2. Bauer A, Oehme S, Geier J (2011) Contact sensitization in the anal and genital area. Curr Probl Dermatol40:133-141 
3. Bauer A, Rodiger C, Greif C et al (2005) Vulvar dermatoses -irritant and allergic contact dermatitis of the vulva. Dermatology 210:143-149

4. Behzad M, MichlC,ArweilerN etal (2014) Lichenoid contact reaction to eugenol presenting as oral lichen planus. Allergo J Int 23:242-245

5. Bircher AJ, Hirsbrunner P, Langauer S (1993) Allergic contact dermatitis of the genitals from rubber additives in condoms. Contact Derm 28:125-126

6. Bork K, Hoede N, Korting GW (1993) Mundschleimhaut- und Lippenkrankheiten. Schattauer, Stuttgart

7. Bork K, Maurer M, Bas M et al (2012) Hereditäres Angioödem durch C1-Inhibitor-Mangel. Allergo J 21:109-118

8. Cicardi M, Aberer W, Banerji A et al (2014) Classification, diagnosis, and approach to treatment for angioedema: consensus report from the Hereditary Angioedema International Working Group. Allergy 69:602-616

9. Craig T, Aygoren-Pursun E, Bork K et al (2012) WAO Guideline for the Management of Hereditary Angioedema. World Allergy Organ J 5:182-199

10. Eubel J, Diepgen TL, Weisshaar E (2015) Allergien im Genitalbereich. Hautarzt 66:45-52

11. Kleine-Tebbe J, Ackermann-Simon J, Hanf G (2012) Role of allergen-specific immunotherapy (desensitization) for the treatment of allergies in Germany. Bundesgesundheitsblatt Gesundheitsforschung Gesundheitsschutz 55(3):343-350. doi:10.1007/ s00103-011-1433-8

12. KrankeB, Trummer M, BrabekEetal (2006) Etiologic and causative factors in perianal dermatitis: results of a prospective study in 126 patients. Wien Klin Wochenschr 118:90-94

13. Kugler K, Brinkmeier T, Frosch PJ et al (2005) Anogenital dermatoses - allergic and irritative causative factors. Analysis of IVDK data and review of the literature. J Dtsch Dermatol Ges 3:979-986

14. Mockenhaupt $M$ (2014) Severe drug-induced skin reactions. Stevens-Johnson syndrome and toxic epidermal necrolysis. Hautarzt 65:415-423

15. Mockenhaupt M, Liss Y (2010) Schwere bullöse arzneimittelinduzierte Hautreaktionen. Aktuell Dermatol 36:1-12

16. Muraro A, Roberts G, Worm M et al (2014) Anaphylaxis: guidelines from the European Academy of Allergy and Clinical Immunology. Allergy 69:1026-1045

17. Ozkaya-Bayazit E (2003) Specific site involvement in fixed drug eruption. J Am Acad Dermatol 49:1003-1007

18. Ozkaya $E$ (2008) Fixed drug eruption: state of the art. JDtsch Dermatol Ges 6:181-188

19. Ozkaya $E$ (2013) Oral mucosal fixed drug eruption: characteristics and differential diagnosis. J Am Acad Dermatol 69:e51-e58

20. Pandhi RK, Kumar AS, Satish DA et al (1984) Fixed drug eruptions on male genitalia: clinical and etiologic study. Sex Transm Dis 11:164-166

21. Price A, Ramachandran S, Smith GP et al (2015) Oral allergy syndrome (pollen-food allergy syndrome). Dermatitis 26:78-88

22. Schlosser BJ (2010) Lichen planus and lichenoid reactions of the oral mucosa. Dermatol Ther 23:251-267

23. Schuster C, Mofarrah R, Aberer W et al (2012) Pitfalls of patch testing with dental materials. $\mathrm{Br} J$ Dermatol 166:674-675

24. Schwartz RA, Mcdonough PH, Lee BW (2013) Toxic epidermal necrolysis: Part I. Introduction, history, classification, clinical features, systemic manifes- tations, etiology, and immunopathogenesis. J Am Acad Dermatol 69:173

25. Sublett JW, Bernstein JA (2011) Seminal plasma hypersensitivity reactions: an updated review. Mt Sinai J Med 78:803-809

26. Torgerson RR, Davis MD, Bruce AJ et al (2007) Contactallergy in oral disease. J Am Acad Dermatol 57:315-321

27. Trautmann A, Kleine-Tebbe J (2013) Allergologie in Klinikund Praxis. Thieme, Stuttgart

28. Valeyrie-Allanore L, Wolkenstein P, Brochard L et al (2010) Open trial of ciclosporin treatment for Stevens-Johnson syndrome and toxic epidermal necrolysis. Br J Dermatol 163:847-853

29. Wedi B, Wieczorek D, Raap U et al (2014) Urticaria. JDtsch Dermatol Ges 12:997-1007

30. Worm M, Jappe U, Kleine-Tebbe J et al (2014) Food allergies resulting from immunological crossreactivity with inhalant allergens: guidelines from the German Society for Allergology and Clinical Immunology (DGAKI), the German Dermatology Society (DDG), the Association of German Allergologists (AeDA) and the Society for Pediatric Allergology and Environmental Medicine (GPA). Allergo JInt 23:1-16

31. Zuberbier T, Aberer W, Asero R et al (2014) The EAACI/GA(2) LEN/EDF/WAO Guideline for the definition, classification, diagnosis, and management of urticaria: the 2013 revision and update. Allergy 69:868-887

\section{DGI plädiert für verbesserte infektiologische Versorgung}

Bei der Behandlung von Krankenhausinfektionen macht die Ausbildung der Ärzte den Unterschied. Dabei kommt Infektiologen eine Schlüsselrolle zu, zeigt eine Auswertung von mehreren internationalen Studien. Die Deutsche Gesellschaft für Infektiologie (DGI) nimmt die Ergebnisse zum Anlass, auf Defizite in der infektiologischen Versorgung und Ausbildung in Deutschland hinzuweisen. Noch immer seien in vielen deutschen Krankenhäusern weder Infektiologen beschäftigt noch infektiologische Konsiliardienste etabliert. Wenn die Eindämmung von Resistenzen und schweren Infektionen gelingen soll, müsse die regelhafte Einbindung von Infektiologen ein selbstverständlicher Bestandteil medizinischer Versorgung werden, betont die DGI. So hat sich in der Metaanalyse bei einer durch Staphylococcus aureus ausgelösten Sepsis der Vorteil einer infektiologischen Beratung deutlich gezeigt: Hier konnte die 30-Tages-Sterblichkeit der Patienten um 40 bis 50 Prozent gesenkt werden. Andere Beispiele seien etwa Patienten mit Herzklappenentzündung (Endokarditis), Organ-Transplantierte oder Intensiv-Patienten.

Drei Aspekte sind demnach für die Behandlungsergebnisse entscheidend: dass ein ausgewiesener Infektiologe zu Rate gezogen wird, dass dies früh geschieht, und dass dieser sich persönlich am Krankenbett ein Bild macht. Ein ausschließlich telefonisches Konsil brachte den Patienten keinen signifikanten Vorteil.

Quelle: Deutsche Gesellschaft für Infektiologie (DGI) www.dgi.de

basierend auf:

Infection (2016) online: 23 Feb. 